\title{
Virtual microscopy, data management and image analysis in Aperio ScanScope system
}

\section{Wojciech Staniszewski}

Precoptic Co.

\begin{abstract}
The histology and the pathology clinical practice undergo a digital revolution. Essential change in laboratory practice - from classical light microscopes, thousands of glass specimens waiting on plates to a virtual microscope and onscreen diagnosis is right now. Currently there are more than 30 different systems for the Virtual Microscopy available on the market. However none of them is so oriented for the practical matters as Aperio ScanScope system.
\end{abstract}

Keywords: telemicroscopy, telepathology, Aperio, Nikon, digital microscopy.

\section{Introduction}

The digital pathology ensures a good quality images which are fundamental for any following diagnosis or research. Also high throughput applications (like massive screening of specimens) require fast and reliable systems for data collection and management connected with algorithms for automated image analysis. It is obvious that traditional microscope combined with camera does not meet these requirements. Even a motorized microscope is too slow in digitalization of slides. Only completely new, designed from scratch slide scanning systems are ready for the task.

An American Company - Aperio, has designed a new generation of ultrafast and reliable scanners - the ScanScope family. Each scanner of this family offers superb scanned image quality, together with a great speed of image acquisition. A powerful databases with image analysis software focused on a diagnostics are available for every imaging system. This is what the pathologists really need, high speed, reliability and high throughput in one device.

\section{Aperio's ScanScope hardware}

Success in the digital pathology depends upon easy and fast creation of the superb quality of the virtual slides (the digitalized glass slides). Aperio's ScanScope devices scan entire surface of glass slides and

Correspondence: W. Staniszewski, Precoptic Co., Arkuszowa

Str. 60, 01-934 Warszawa, Polska; (+4822) 8341225,

e-mail: wojciech.staniszewski@precoptic.pl create digital copies of them in few minutes. To be precise -2 minutes if region of interest size is 15 to 15 millimeters.

All ScanScopes use very special, patented, linear cameras for digitalization of the glass slides. Those cameras in combination with the highest class Nikon's PLAN APO objectives, gives the best quality of image on the market.

Among all ScanScopes available on the market we can find different equipment dedicated for different users depending on the system throughput requirements. Despite those differences, all ScanScopes shares the same scanning procedure.

ScanScope GL - is a single slide model is capable of manual loading and scanning only one slide at a time. It was designed for a small research and education facilities in order to fully use of the virtual slides. The GL model has the same quality of optics and cameras as higher models of ScanScope - CS and XT. What is also important GL can be equipped with full Spectrum ${ }^{\circledR}$ PLUS data storage and analysis software. At $20 \times / 0.75$ Plan Apo objective scans $15 \mathrm{~mm} \times 15 \mathrm{~mm}$ slide area in under of 2 minutes. The scanning magnifications are: $20 \times, 40 \times(20 \times$ objective with $2 \times$ magnification changer). The scanning magnification - resolution - 20×: $0.50 \mu \mathrm{m} /$ pixel, 40×: $0.25 \mu \mathrm{m} /$ pixel. Please also pay attention to numerical aperture of this objective. This parameter is highly connected to objective resolution and ability to see minute structures. Factor 0.75 means that objective $20 \times$ from ScanScope has better ability to see details of specimen than typical objective $60 \times$. 
ScanScope CS - is a five slides model. It scans five slides one by one automatically loading them from a tray. This is a multipurpose system for medium size facilities. Auto loading saves significant amount of time of the lab staff. It scans $15 \mathrm{~mm} \times 15 \mathrm{~mm}$ slide area with $20 \times / 0.75$ Plan Apo objective in less than 2 minutes. Five slides about this size will be scanned from 10 up to 15 minutes, and this is enough time for Scanscope operator to do other useful work.

Glass slide dimensions are: $1 \times 3$ " $(25 \times 75 \mathrm{~mm})$ slides, $2 \times 3$ " $(50 \times 75 \mathrm{~mm})$. Scanning magnification: $20 \times, 40 \times(20 \times$ with $2 \times$ magnification changer $)$. Resolution at $20 \times: 0.50 \mu \mathrm{m} /$ pixel, $40 \times: 0.25 \mu \mathrm{m} /$ pixel

ScanScope XT - One hundred and twenty slides model, designed for high throughput applications. XT offers even higher speed of scanning of a single specimen.

At $20 \times$ objective it scans $15 \mathrm{~mm} \times 15 \mathrm{~mm}$ slide area in less than 2 minutes. This speed and automatic loader for 120 specimens clearly show the extreme capabilities of XT scanner. Single XT unit can be a core of a large histology department of a hospital or pharmaceutical company. No other system available on the market can do this. ScanScope XT is also capable for scanning $2 \times 3$ " slides.

Scanning magnification: $20 \times, 40 \times(20 \times$ with $2 \times$ magnification changer). Resolution at $20 \times: 0.50 \mu \mathrm{m} /$ pixel, $40 \times: 0.25 \mu \mathrm{m} /$ pixel

Scanscope OS - One slide, high magnification model with immersion oil objective. Designed for hematologists and microbiologists. Offers magnification 100x and 50x producing unbelievable resolution of 0.14 $\mu \mathrm{m} /$ pixel at 100x and $0.28 \mu \mathrm{m} /$ pixel at 50x. ScanScope OS can easily scan an area of $10 \times 10 \mathrm{~mm}$ in approximately 20 minutes.

Every scanner allows user to perform an automatic scanning. The user just puts the slide on a tray and presses green button on the device. Scanner will search the glass for the specimen, then will set the area for scanning. After that The ScanScope automatically decides for spots of autofocusing and white balance. When everything is ready the scans begins. After scanning a digital copy of scanned specimen is being sent to Spectrum ${ }^{\circledR}$ database and becomes instantly available in the Internet website (of course if it is allowed by user). There is an option which allows to scan manually also. User has the full control over each stage. During manual scan it is possible to switch to auto mode on every stage.

\section{Aperio's ScanScope software}

Huge amount of data generated by the scanners has to be stored, analyzed and presented to the end user (the clinician). To accomplish that every ScanScope operates with Aperio's Spectrum software which provides providing a functionality for viewing, editing, and the basic search and digital slide management. Also all scanners can be connected to any institute internal network (or the Internet) for the patient data management and workflow control.

Due to very high image resolution the size of an average virtual slide is about $300 \mathrm{MB}$ (mega bytes). But this may significantly vary from few megabytes to terabytes per slide depending on scanned area and magnification. The bigger area and higher magnification the larger file will be. Such big files create instantly two problems: one with storage, and second with sharing through the Internet. The Aperio designers solved this by lossless compression of the images. This saves space and keeps the image quality at the same, the highest level. A few options are available but the most effective compression is JPEG2000. JPEG2000 can compress an image at least 4 times.

The second problem is the slide sharing over the Internet. It is possible and comfortable for end user to view 1 TB (terra byte) slides through relatively slow Internet connection of $64 \mathrm{~KB} / \mathrm{sec}$ (kilo byte) per second. How it is possible? Everything is based on a simple observation and the special computer algorithms. If a user is watching specimen under $100 \times$ magnification, in fact he is not watching entire specimen but only a tiny spot. The digital information about a tiny spot is tiny. So no high speed connection is needed. If the user moves to another spot, a new information is being sent. To make the viewing faster software anticipate next user move and downloads a new part of image in advance. To facilitate viewing the slides ImageScope software was released. ImageScope is freely available at Aperio web site. It has all the functions required for viewing slides and their measurements. User can compare multiple slides, side by side, on the screen, change magnifications and spots on the specimen. There is also another way of viewing slides which does not requires installation of ImageScope. Every database can present images through so called web viewer which utilizes Flash technology - present in every web viewer like Firefox or MS Internet Explorer browser. Viewing slides in this way is just as easy as opening the web page.

ImageScope can be also a platform for telepathology. The user can consult the image over the Internet with 30 persons at the same time: other specialists or students. During such consultation or lecture each person sees the same specimen, the same place and magnification as the teleconference leader. The conference leader can choose specimens, draw marks or arrows on the specimen visible for all participants. It is possible to show even the mouse cursor movements. During the 
conference it is possible to appoint a new leader for example to ask a student what he has understood.

ImageScope also can be a platform for image analysis.

\section{Image analysis}

Spectrum ${ }^{\circledR}$ database is powerful image analysis software. The simplest algorithm Positive Pixel Count is for general densitometry measurements. It gives information about number of pixels and optical density of staining as a result. More advanced algorithms are for nuclear and membrane evaluation. Those algorithms analyze not only the optical density but also some morphological factors. For example the membrane algorithm used for HER2 analysis measures the optical density and continuity of membrane staining. The color deconvolution algorithm can separate different staining performed on the same specimen which allows the further analysis with other algorithms.

Histology Pattern Recognition software - GENIE is unique. It can find any pattern on histological specimen. GENIE can learn what user wants to find and finds it analyzing morphology and staining of cells. GENIE can be used for searching of the tumor cells, blood vessels or any other interesting objects in a specimen. Areas found and marked by GENIE can by analyzed with other algorithms.

All available algorithms are stored on the server. User can launch algorithm locally on his computer or on a server. It is worth to mention that calculations made on server are much faster than those on the personal computers. Also by launching calculations on the server it is possible to order an analysis of hundreds specimens as overnight work.

\section{Summary}

Aperio ScanScope scanners are technically really useful in a everyday work and widely accepted by the people on the field of the histology and the pathomorphology [3]. Over 500 hundred units sold (most of them in last two years) means a lot. More than twothirds of the top 15 rated US hospitals and two-thirds of the top 15 pharmaceutical companies utilizes Aperio equipment.

Pathology goes digital with Aperio Scanscope!

\section{References}

[1] Rojo MG, García GB, Mateos CP, García JG, Vicente MC. Critical comparison of 31 commercially available digital slide systems in pathology. Int J Surg Pathol. 2006;14(4):285-305.

[2] Aperio company web site. Available at: http://www.aperio.com. Accessed June 17, 2009.

[ 3] Słodkowska J, Chyczewski L, Wojciechowski M. Virtual slides: application in pulmonary pathology consultations. Folia Histochem Cytobiol. 2008;46(1):121-4.

Submitted: 16 June, 2009 Accepted after revision: 20 September, 2009 